\title{
Vigilancia nacional de aislamientos clínicos de Enterococcus faecalis resistentes al linezolid portadores del gen optrA en Colombia, 2014-2019
}

\author{
Sandra Yamile Saavedra1, Johan Fabian Bernal', Efrain Montilla-Escudero', German Torres , \\ Mabel Karina Rodríguez ${ }^{1}$, Andrea Melissa Hidalgo ${ }^{1}$, María Victoria Ovalle ${ }^{1}$, Sandra Rivera $^{3}$, \\ Enrique Perez-Gutierrez ${ }^{4}$ y Carolina Duarte ${ }^{1}$
}

Forma de citar

Saavedra SY, Bernal JF, Montilla-Escudero E, Torres G, Rodríguez MK, Hidalgo AM, et al. Vigilancia nacional de aislamientos clínicos de Enterococcus faecalis resistentes al linezolid portadores del gen optrA en Colombia, 2014-2019. Rev Panam Salud Publica. 2020;44:e104. https://doi.org/10.26633/RPSP.2020.104

RESUMEN Objetivo. Describir las características epidemiológicas, fenotípicas y genéticas de aislamientos clínicos por-
tadores de optrA identificados en la vigilancia de resistencia antimicrobiana por el laboratorio del Instituto
Nacional de Salud de Colombia.
Métodos. Entre octubre de 2014 y febrero 2019 , se recibieron 25 aislamientos de Enterococcus spp. resis-
tentes al linezolid. La identificación y sensibilidad antimicrobiana se determinó con Vitek 2 y la concentración
inhibitoria mínima (CIM) al linezolid se estableció con E-test. El gen optrA se detectó mediante PCR. La
diversidad genética de aislamientos positivos para optrA se analizó con Diversilab ${ }^{\circledR}$. Se seleccionaron seis
aislamientos para llevar a cabo la secuenciación del genoma completo.
Resultados. Se confirmó el gen optrA en $23 / 25$ aislamientos de E. faecalis de siete departamentos de Colom-
bia. Los aislamientos presentaron una CIM al linezolid entre 8 y $>256 \mu g / m L$. La tipificación por Diversilab ${ }^{\circledR}$
indicó una amplia variabilidad genética. Todos los aislamientos analizados mediante secuenciación del
genoma completo, presentaron genes de resistencia fexA, ermB, IsaA, tet(M), tet(L) y dfrG además de optrA
y fueron negativos para otros mecanismos de resistencia al linezolid. Se identificaron tres secuencias tipos
y tres variantes de optrA: ST16 (optrA-2), ST476 (optrA-5) y ST618 (optrA-6). El entorno genético de los ais-
lamientos optrA-2 (ST16) presentó el segmento impB, fex, optrA, asociado a plásmido, mientras que en dos
aislamientos (optrA-6 y optrA-5) se encontró el elemento cromosómico transferible Tn6674-like.
Conclusión. Los aislamientos clínicos positivos para optrA presentan una alta diversidad genética, con diferentes
clones y variantes de optrA relacionados con dos tipos de estructuras y diferentes elementos genéticos móviles.

Palabras clave

Enterococcus faecalis; linezolid; farmacorresistencia microbiana; Colombia.

El linezolid es un antimicrobiano de la familia de las oxazolidinonas usado como tratamiento de infecciones causadas por bacterias Gram positivas, especialmente para microorganismos multidrogorresistentes como Staphylococcus aureus resistente

1 Grupo de Microbiología, Dirección Redes en Salud Pública. Instituto Nacional de Salud (INS), Bogotá, Colombia. Correo electrónico: cduarte@ins.gov.co

2 Grupo de resistencia antimicrobiana, Centro de investigación Tibaitata corporación colombiana de investigación agropecuaria (AGROSAVIA), Bogotá, Colombia a la meticilina (SARM) y Enterococcus resistente a la vancomicina (ERV) (1-3). De acuerdo con la Organización Mundial de la Salud, las oxazolidinonas hacen parte de la lista de antimicrobianos de importancia crítica para la salud de los seres 
humanos (https://www.who.int/foodsafety/publications/cia 2017es.pdf?ua=1.AUTOR) .

Aunque la frecuencia de resistencia al linezolid en Enterococcus spp. a nivel mundial es baja $(<1,2 \%)$ según lo notificado por programas como SENTRY (Antimicrobial Surveillance Program, 0,38\% para el período 2008 a 2016), LEADER (Linezolid Experience and Accurate Determination of Resistance, 0,5\% entre 2011 y 2015) y ZAAPS (Zyvox® Annual Appraisal of Potency and Spectrum, 1,17\%), en los últimos años a nivel mundial se ha observado un incremento en los reportes de resistencia al linezolid por diferentes mecanismos (4-8).

La resistencia al linezolid se ha asociado con: i) mutaciones cromosómicas del gen $A R N r 23 S$ y las proteínas ribosomales L3, L4 y L22 (1, 2), y ii) genes transferibles en plásmidos como cfr (9), poxtA (10) y optrA (11). El gen cfr codifica una metiltransferasa ARNr $23 S$ que confiere resistencia a las oxazolidinonas (linezolid), fenicoles, lincosamidas, pleuromutilinas y estreptograminas A (9), mientras que los genes poxtA y optrA codifican una proteína transportadora tipo ATP-binding cassette (ABC)-F que confiere resistencia a las oxazolidinonas (linezolid y tedizolid) y fenicoles a través de un mecanismo de protección del ribosoma (12).

El gen optrA fue identificado por primera vez en China en 2015 (11); se ha detectado principalmente en diferentes especies de Enterococcus spp., (4, 11-14), Staphylococcus sciuri, Staphylococcus simulans (13) y Streptoccosus suis (15). Así mismo, se han identificado microorganismos portadores del gen optrA en alimentos de origen animal $(11,16-18)$, personas hospitalizadas e individuos sanos (4, 7, 11-14), aguas residuales (19) y suelos (20). Este gen ha sido detectado en diferentes plásmidos y cromosomas $(4,11,13-21)$. Actualmente optrA se ha reportado en países de Asia $(4,14,15,19,21)$, Europa $(4,8,17,22-24)$, África $(18,19,21)$ y América $(4,16,21)$. En Colombia, la primera detección de optrA se realizó en Enterococcus faecalis recuperados en carne cruda de origen avícola (16).

El objetivo de este estudio es describir las características epidemiológicas, fenotípicas y genéticas de aislamientos clínicos portadores del gen $\operatorname{optr} A$, recuperados a través de la Vigilancia Nacional por Laboratorio de la Resistencia a los Antimicrobianos, liderada por el Grupo de Microbiología de la Dirección de Redes en Salud Pública del Instituto Nacional de Salud (INS) de Colombia.

\section{MATERIALES Y MÉTODOS}

Se realizó un estudio retrospectivo, descriptivo, transversal. Entre octubre de 2014 y febrero de 2019, la vigilancia nacional por laboratorio de la resistencia a los antimicrobianos en microorganismos causantes de infecciones asociadas a la atención en salud (IAAS) del INS recibió 25 aislamientos de Enterococcus spp. (uno en 2014, dos en 2016, cuatro en 2017, 14 en 2018 y cuatro entre enero y febrero de 2019) resistentes al linezolid, enviados por 12 instituciones desde 7 departamentos del país de manera voluntaria. La confirmación del género y la especie bacterianas y de la susceptibilidad antimicrobiana se realizó a través del sistema automatizado Vitek-2 Compact $^{\circledR}$ (bioMérieux), y se determinó la concentración inhibitoria mínima (CIM) al linezolid usando el método epsilométrico E-test ${ }^{\circledR}$ (bioMérieux, Durham, EE.UU.). Los resultados de susceptibilidad fueron interpretados de acuerdo a los parámetros del Clinical and Laboratory Standards Institute (25).

El ADN de los aislamientos se obtuvo por lisis celular con ebullición. La detección del gen optrA se realizó por PCR convencional, usando los iniciadores y las condiciones descritas por Wang et al (11).

Los aislamientos positivos para optrA fueron tipificados a través de rep-PCR automatizada por electroforesis capilar, utilizando el sistema Diversilab (bioMérieux, Marcy l'Etoile, Francia) kit Enterococcus, siguiendo las instrucciones del fabricante. Los patrones electroforéticos fueron analizados mediante el software en línea de Diversilab ${ }^{\circledR}$, usando el coeficiente de correlación de Pearson. Los aislamientos con similitud $\geq 97 \%$ se consideraron indistinguibles, $\geq 95 \%$ a $<97 \%$ aislamientos similares y $<95 \%$ aislamientos diferentes.

Se seleccionaron seis aislamientos positivos para optrA para realizar secuenciación del genoma completo (SGC). La extracción de ADN genómico se realizó a partir de colonia en agar BHI usando PureLink ${ }^{\mathrm{TM}}$ Genomic DNA mini Kit (ThermoFisher Scientific, Massachusetts, EE.UU.) de acuerdo con las instrucciones del fabricante. El ADN se cuantificó usando Qubit 4 (ThermoFisher Scientific, Massachusetts, EE.UU.) y se verificó la calidad e integridad con Nanodrop (ThermoFisher Scientific, Massachusetts, EE.UU.) y gel de agarosa. Las librerías indexadas de ADN se elaboraron usando los kits Nextera XT y Dual index de Illumina, siguiendo las especificaciones del fabricante (Illumina, San Diego, EE.UU.). El control de calidad de las librerías (tamaño y concentración) se realizó usando Qubit 4 y el estuche High Sensitivity DNA para Bioanalyzer 2100 (Agilent, Santa Clara, EE.UU.). Las librerías genómicas fueron secuenciadas usando kit V2 de 500 ciclos en la plataforma Illumina Miseq (Illumina, San Diego, EE.UU.). La calidad de las lecturas de las secuencias se verificó mediante el software FASTQC (Babraham Bioinformatics, https://www. bioinformatics.babraham.ac.uk/projects/fastqc/).

La presencia de genes de resistencia y elementos móviles se analizó usando los datos genómicos generados y comparándolos con las bases de datos de ResFinder (26), LRE-Finder (27) y Plasmidfinder (28). La tipificación molecular se realizó con MLST y el llamado de SNPs usando CSIphylogeny, todos softwares de libre acceso del Center of Genomic Epidemiology, DTU-Denmark (http://www.genomicepidemiology.org/). El árbol filogenético y los metadatos se visualizaron en la aplicación Microreact. El ambiente genético fue caracterizado y comparado usando la anotación de PROKKA (29) y Genbank, los alineamientos de los clusters del gen optrA y las referencias seleccionadas usando BLAST-N (30), y los softwares Artemis 18.0.3 y ACT 18.0.3 (31) del instituto Sanger-UK.

Para la investigación de campo se priorizó el departamento con el mayor número de casos; se caracterizaron en total nueve casos. Se diseñó una encuesta para recopilar las variables sociales y demográficas, antecedentes clínicos, familiares, laborales y de alimentación. La información se analizó en Epi info ${ }^{\mathrm{TM}} 7$.

Este estudio no requirió aprobación por un comité de ética por encontrarse en el contexto de la vigilancia en salud pública. Los datos demográficos de los pacientes se anonimizaron y la información recolectada en campo contó con consentimiento informado firmado por cada individuo. Todos los datos fueron manejados bajo protección de confidencialidad (ley 1581 de 2012 de Colombia).

\section{RESULTADOS}

De los 25 aislamientos recibidos, se confirmó la presencia de optrA en 23 (92\%) Enterococcus faecalis (cuadro 1) de siete departamentos (Antioquia, Bogotá D.C., Casanare, Cesar, Norte de 


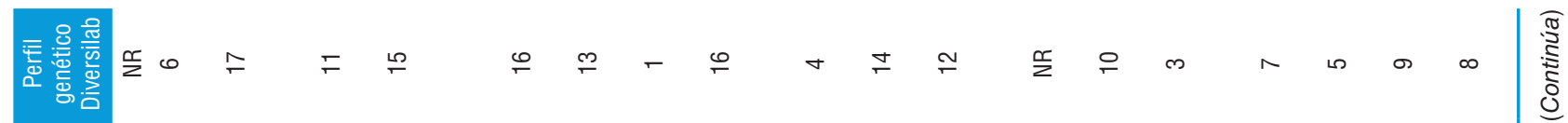

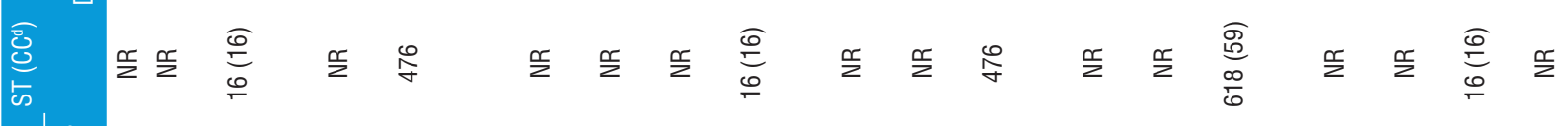

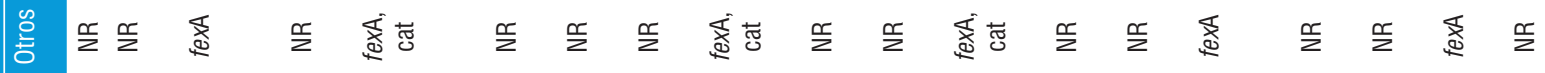

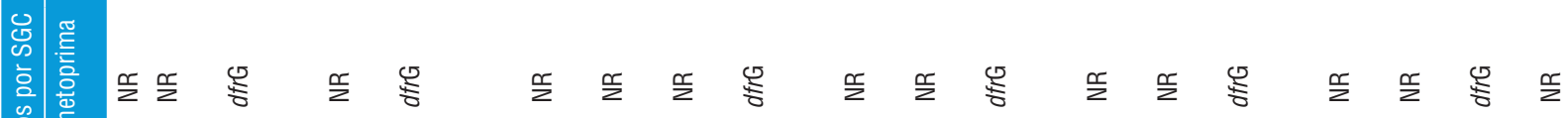

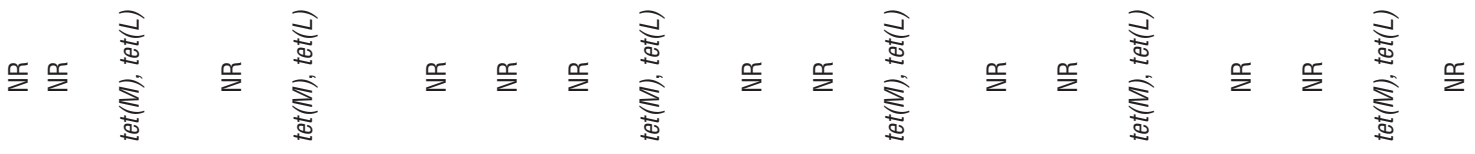

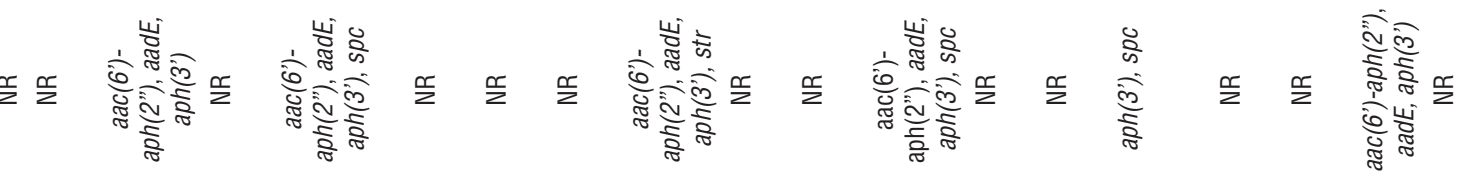

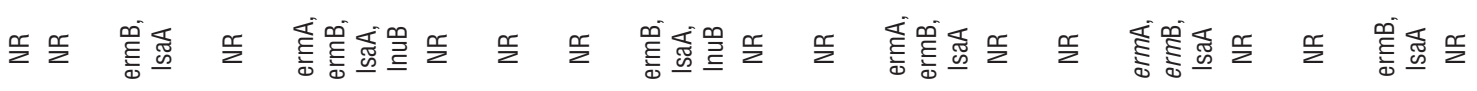

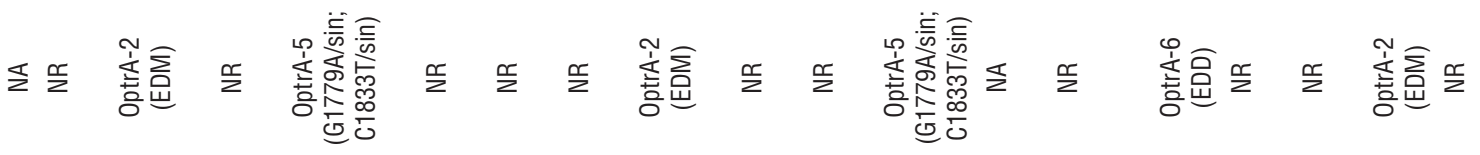

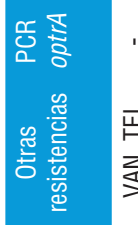

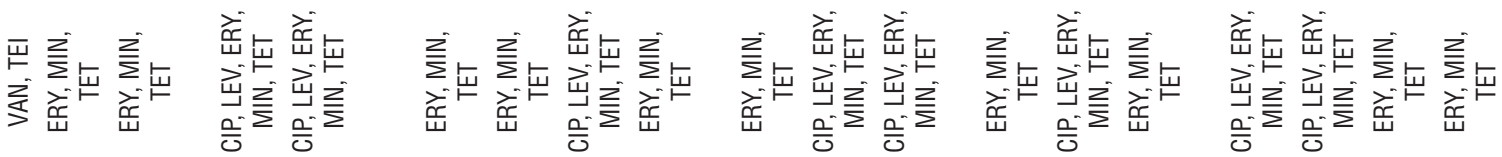

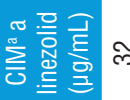

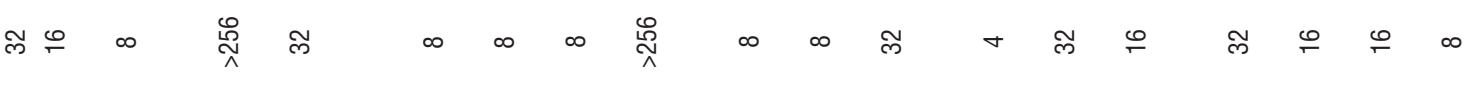

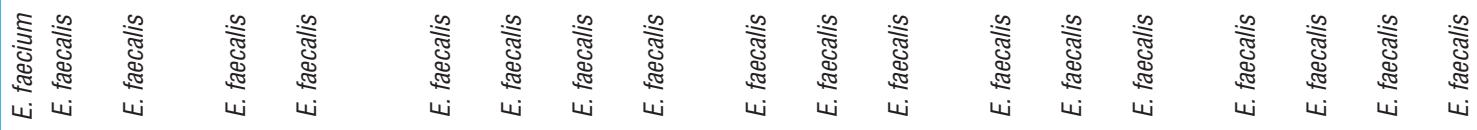

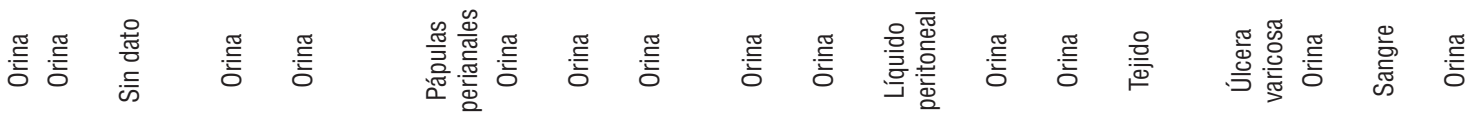

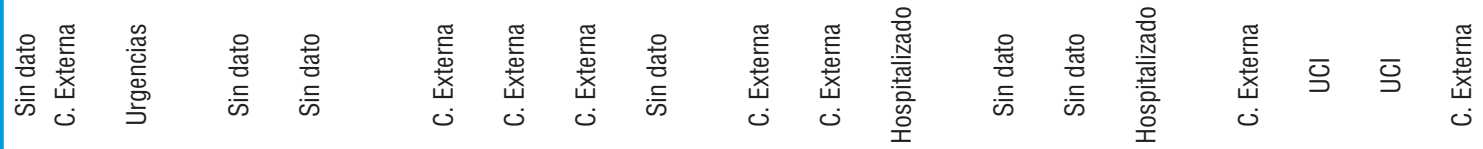

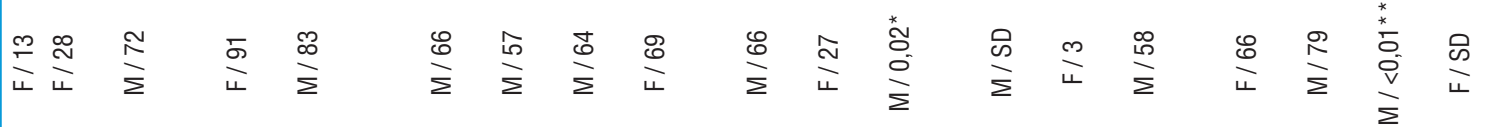

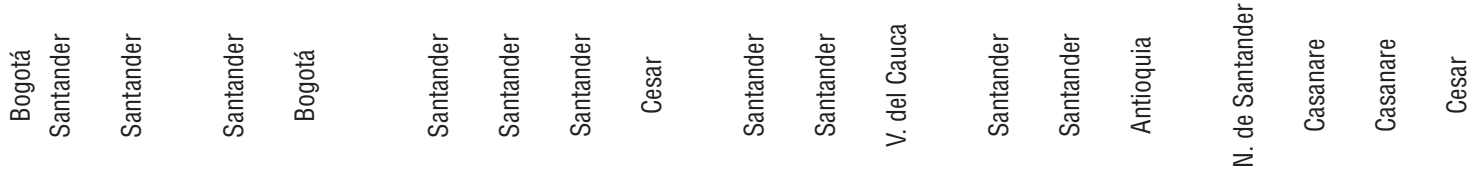

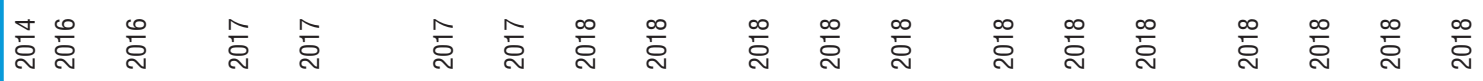

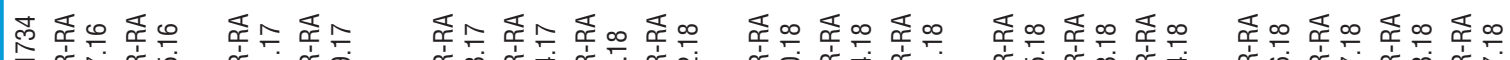

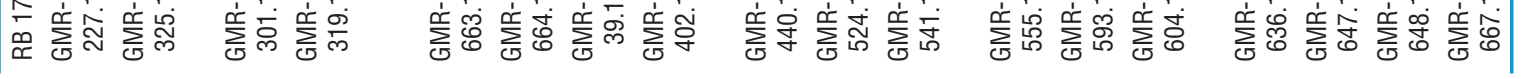




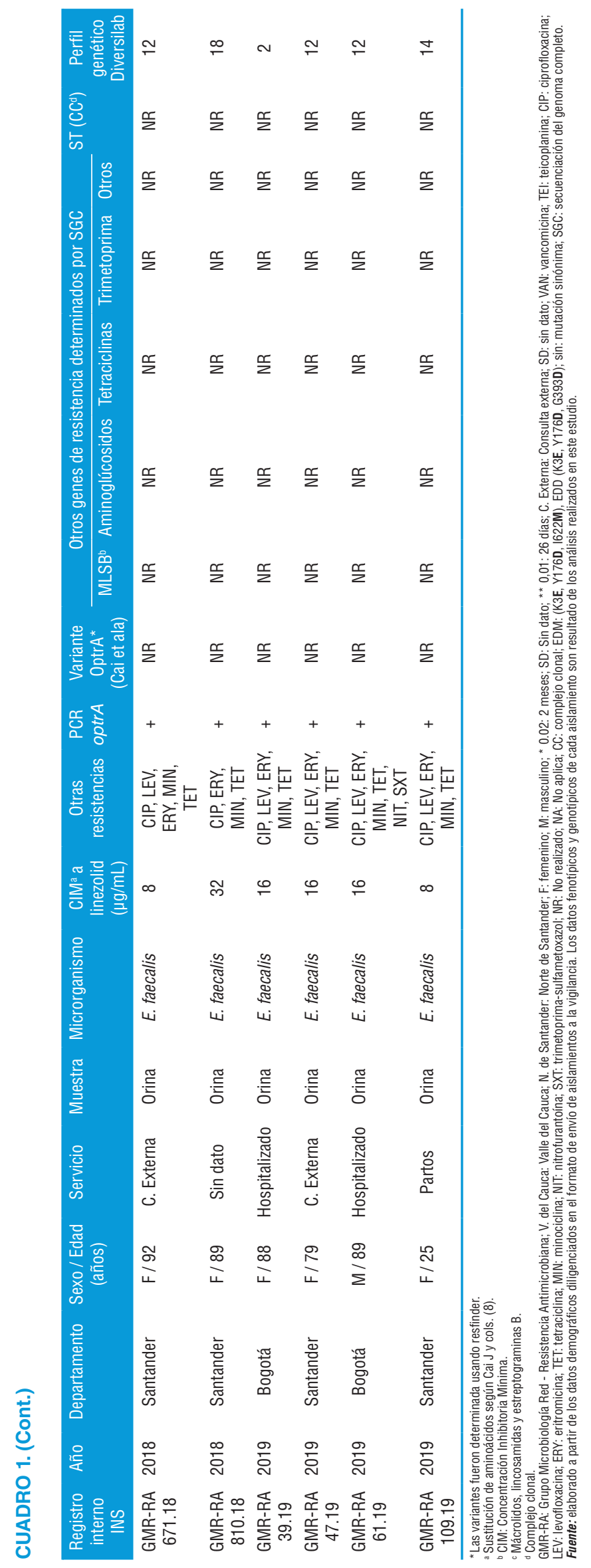


Santander, Santander y Valle del Cauca) y 10 instituciones. La gran mayoría ( $n=13 ; 56,5 \%)$ de pacientes fueron procedentes del departamento de Santander (cuadro 1). Del total de pacientes, $10(43,5 \%)$ asistieron a consulta externa (servicio ambulatorio), ocho $(34,8 \%)$ se encontraban hospitalizados y de los restantes no se obtuvo información (cuadro 1). La edad de los pacientes presentó un rango de entre 26 días y 91 años. La mayoría $(\mathrm{n}=14$; $60,9 \%$ ) fueron adultos mayores (>60 años); el 52.2\% ( $n=12)$ corresponden a mujeres y la muestra de la que se aisló $E$. faecalis portador de optrA con más frecuencia fue orina $(n=17 ; 73,9 \%)$. De los tres casos identificados en la población pediátrica, dos casos (uno de 26 días y otro de dos meses) corresponden a pacientes hospitalizados desde su nacimiento (cuadro 1).

\section{Perfil de susceptibilidad antimicrobiana}

Todos los aislamientos optrA positivos fueron resistentes al linezolid, con una CIM entre $8 \mu \mathrm{g} / \mathrm{mL}$ y $>256 \mu \mathrm{g} / \mathrm{mL}$ (cuadro 1). También fueron resistentes a la eritromicina, la minociclina y la tetraciclina. El 60,9\% (n=14) fueron resistentes a la ciprofloxacina. Todas las muestras fueron sensibles a la ampicilina, la teicoplanina y la vancomicina

\section{Diversidad genética de los aislamientos portadores de optrA}

Los aislamientos de E. faecalis portadores de optrA se clasificaron en 17 patrones electroforéticos (similitud $<65 \%$ ), divididos en 14 perfiles únicos y tres grupos genómicos (figura 1): grupo 12, formado por cuatro aislamientos (GMR RA-671.18, GMR
RA-541.18, GMR RA-47.19 y GMR RA-61.19) con similitud de 97,8\% (indistinguibles entre sí), enviados de Santander, Valle del Cauca y Bogotá (figura 1 y cuadro 1); grupo 14, formado por GMR RA-524.18 y GMR RA-109.19, con similitud de 95,5\%; y grupo 16 formado por GMR RA-402.18, GMR RA-663.17 y GMR RA-325.16 con similitud de 95,9\%, recuperados en Santander y Cesar (figura 1 y cuadro 1).

\section{Secuenciación del genoma completo y distribución de genes de resistencia}

En los seis aislamientos analizados por secuenciación del genoma completo se identificaron tres variantes de optrA, optrA-2 "variante EDM" $(\mathrm{n}=3)$, optrA-5 (n=2) y optrA-6 "variante EDD" (n=1) (cuadro 1) y no se identificaron otros genes asociados con resistencia al linezolid, como genes cfr ó poxtA; tampoco se detectaron mutaciones en ARNr 23S ni en las proteínas ribosomales L3, L4 y L22. En cuanto a otros determinantes de resistencia, se detectó la presencia de diversos genes que codifican resistencia a aminoglucósidos [genes $s p c$, aac $\left(6^{\prime}\right)$ $\operatorname{aph}\left(2^{\prime \prime}\right)$, aph $\left.\left(3^{\prime}\right)\right]$, fenicoles (genes cat, fexA), estreptograminas, macrólidos, lincosamidas $(\operatorname{erm} \mathrm{A}, \operatorname{erm} \mathrm{B}, \operatorname{ls} a \mathrm{~A}, \ln u \mathrm{~B})$, tetraciclinas [genes tet $(M)$ y tet $(L)$ ] y trimetoprima-sulfametoxazol $(d f r G)$. Además de optrA, todos los aislamientos fueron portadores de genes fexA, ermB, lsaA, tet( $M)$, tet $(L)$ y dfrG (cuadro 1).

\section{Ambiente genético del gen optrA}

El ambiente genético de optrA fue comparado in silico con datos previamente publicados en el NCBI. Todos los

FIGURA 1. Dendrograma, imagen del gel virtual y matriz de similitud de aislamientos de Enterococcus faecalis positivos para optrA, tipificados con Diversilab ${ }^{\circledR}$
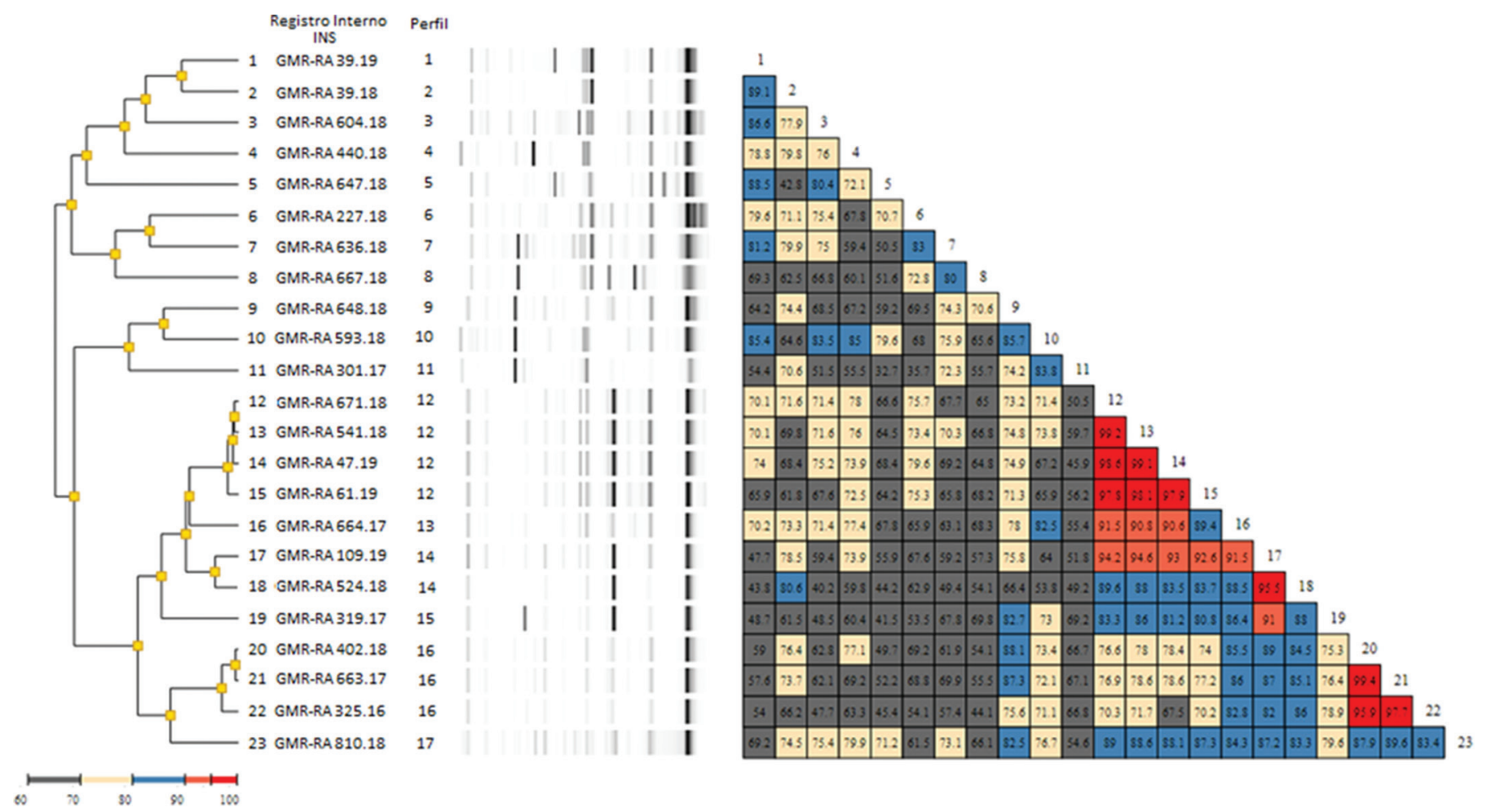

Fuente: elaborada a partir de los patrones electroforéticos obtenidos de la rep-PCR, usando el sistema Diversilab ${ }^{\oplus}$ y las muestras analizadas en este estudio. 
FIGURA 2. Representación esquemática de ambiente genético del gen optrA. Las flechas indican la dirección de la transcripción. a. Representación esquemática de aislamientos portadores de optrA-2 comparados con la cepa pS7316 (LC499744). b. Representación esquemática de aislamientos portadores de optrA-5 y optrA-6, comparados con la plataforma Tn6674 del aislamiento TZ2 (MH225421).

a $\underset{\text { repeat_region }}{\square}$
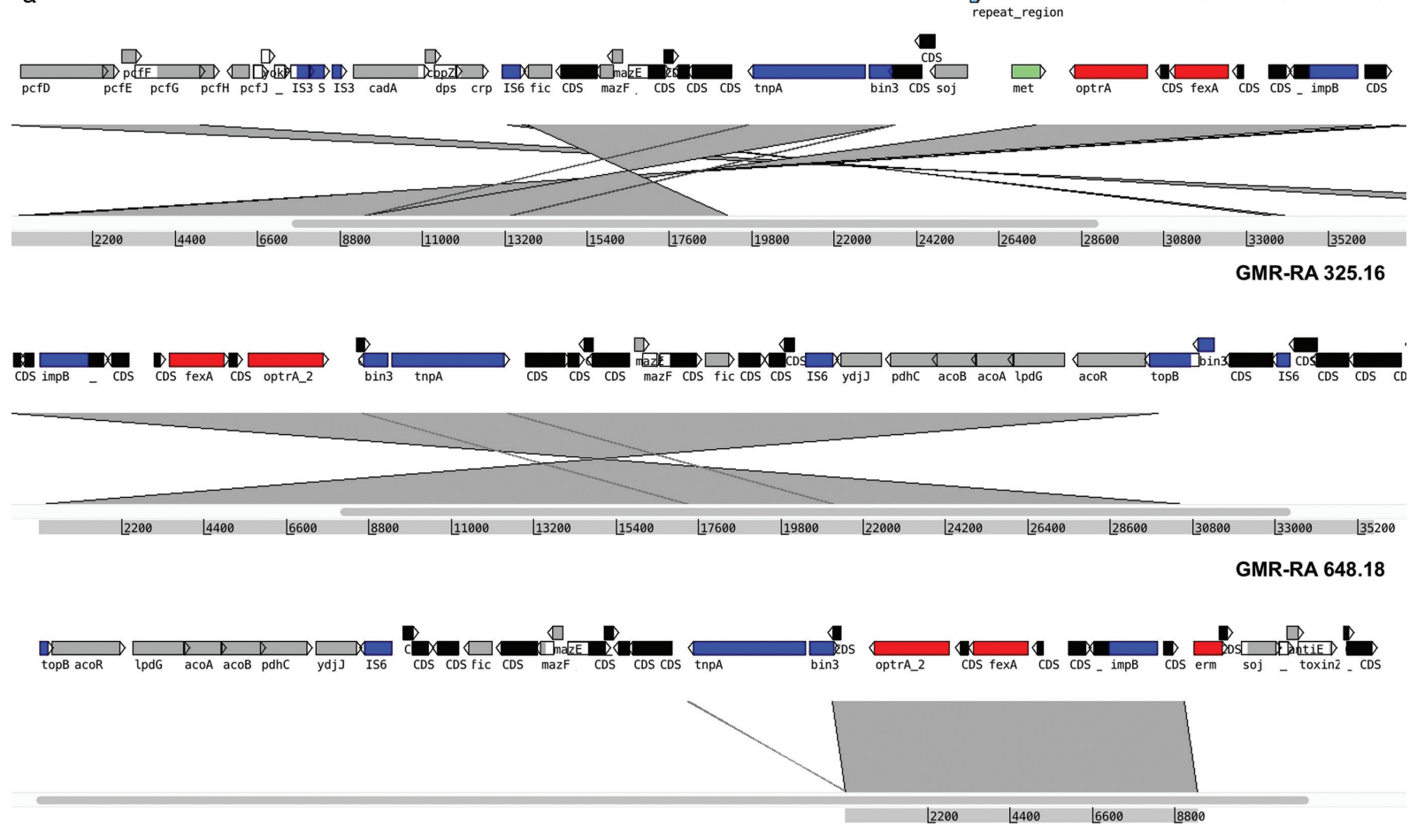

GMR-RA 402.18

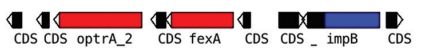

b

TZ2 (MH225421)

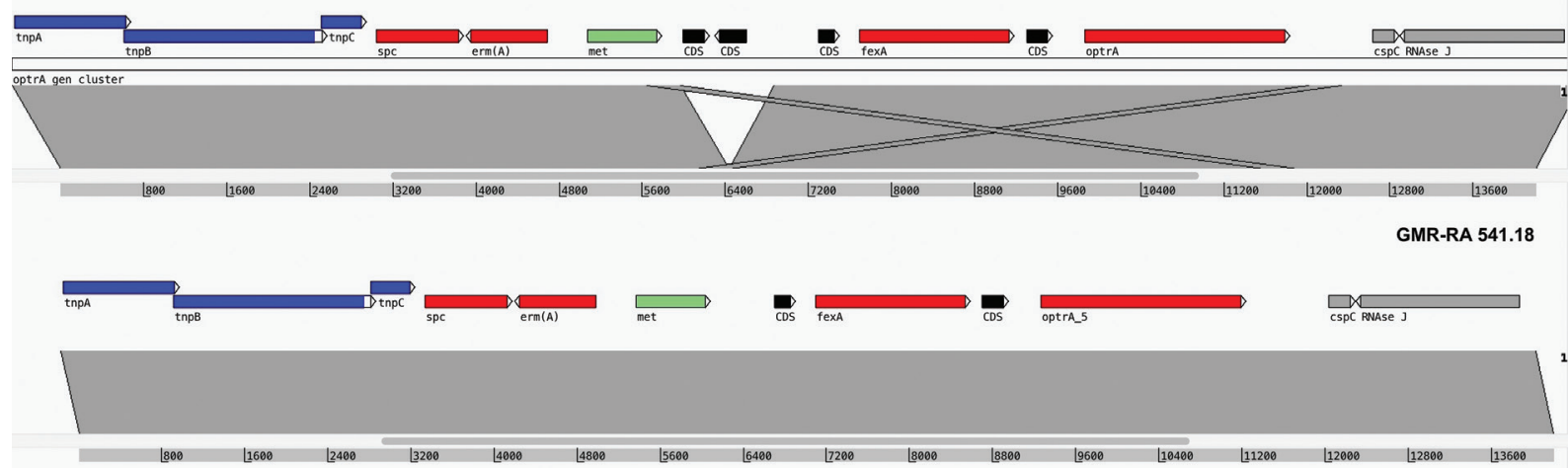

GMR-RA 604.18

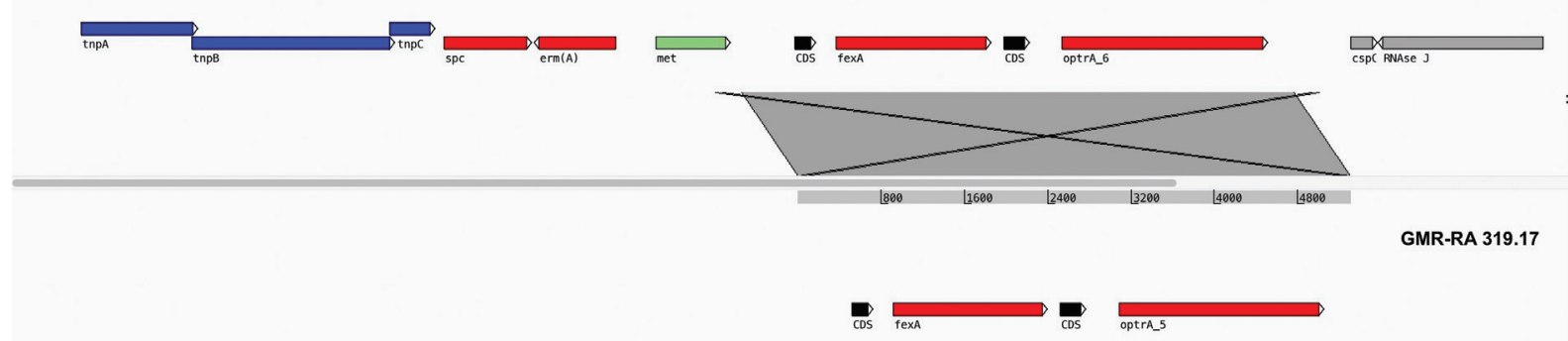

Las flechas rojas representan genes de resistencia antimicrobiana. Las flechas azules representan genes asociados a transposición y recombinación, replicación y reparación. La flecha verde representa el gen met que codifica una metiltransferasa. Las flechas grises representan genes que codifican otras funciones y las flechas negras representan proteínas hipotéticas. 
aislamientos presentaron genes de resistencia fexA y optrA localizados en la misma orientación (figura 2). En el entorno genético de aislamientos portadores de optrA-2 se observó la presencia de un segmento común formado por los genes “imp B (gen de reparación de ADN asociado a plásmido), fexA y optrA"; esta estructura se ha asociado con plásmidos (figura 2a) (19). Además del segmento mencionado, los aislamientos GMR-RA 325.16 y GMR-RA 648.18 mostraron dos genes relacionados con trasposición bin3 (ADN invertasa del Tn552) -y tnpA (transposasa de Tn3), y más alejado de optrA, se detectó un locus que codifica la secuencia de inserción IS6 (figura 2a). En los aislamientos GMR-RA 541.18 (optrA-5) y GMR-RA 604.18 (optrA-6) se identificó el Tn6674-like conformado por ocho locus, distribuidos del modo siguiente: tres genes asociados con transposición (transposasas tnp A - C), cuatro genes de resistencia que fueron optrA, fexA, spc (resistencia a espectinomicina) y erm(A1) y una metiltransferasa (met) (figura 2b).

\section{Análisis fillogenético y distribución geográfica}

Se identificaron tres secuencias tipos, cada una asociada a una variante de optrA; tres aislamientos fueron ST16 "CC16" (optrA-2), dos fueron ST476 (optrA-5) y uno fue ST618 "CC59" (optrA-6) (cuadro 1). El árbol filogenético muestra que los tres aislamientos ST16 se encuentran relacionados genéticamente a pesar de haber sido aislados en diferentes departamentos; una situación similar se observa con los aislamientos ST476 (https://microreact.org/project/UUqRJN0yC/ed3e5466).

\section{Estudio epidemiológico}

Santander fue el departamento con el mayor número de casos (nueve en total provenientes de los municipios de Bucaramanga y Floridablanca, ciudades cercanas). Del total de casos, ocho fueron identificados en consultas ambulatorias, siete fueron clasificados como colonizados y seis casos eran adultos mayores (>60 años). En ninguno de los nueve individuos se identificó exposición previa al linezolid o alguna relación entre el servicio de hospitalización, antecedentes clínicos, familiares, ocupacionales o hábitos de alimentación y consumo.

\section{DISCUSIÓN}

En Colombia, los datos de la vigilancia nacional de resistencia en IAAS, usando el software WHONET5.6, indican que para 2018 la resistencia al linezolid en E. faecium es de 1,7\% ( $n=1512)$ y en E. faecalis de 1,7\% ( $\mathrm{n}=12.051)$; estos datos muestran un leve incremento si se comparan con datos reportados a nivel mundial por diferentes programas de vigilancia como SENTRY, LEADER y ZAAPS (4-7). En nuestro estudio, la prevalencia de optrA en $E$. faecalis resistentes al linezolid fue de $96 \%$ (23); este dato es similar al reportado para 2016 en Alemania, donde la prevalencia de optrA fue del 90,9\% en E. faecalis según lo reportado por el Instituto Robert Koch (centro nacional de referencia en Alemania).

Las variantes optrA-2, optrA-5 y optrA-6 identificadas en este estudio han sido reportadas en humanos $(4,7,8,14,21) \mathrm{y}$ alimentos de origen animal $(18,19,21)$; optrA-2 se ha identificado incluso en aguas residuales $(18,21)$. En Colombia, optrA-2 se ha descrito previamente en carne cruda $(16,21)$, mientras que es la primera vez que se reporta optrA5 y optrA-6. La variante más común fue optrA-2 (EDM) y aunque ninguno de estos aislamientos presentó mecanismos adicionales de resistencia al linezolid, todos fueron resistentes al linezolid con CIM entre 8 y $>256 \mu \mathrm{g} / \mathrm{mL}$, contrastando con los datos publicados por Cai y cols (14) donde aislamientos optrA-2 (EDM) presentaron una $\mathrm{CIM}$ al linezolid en el rango sensible e intermedio ( $\leq 2$ y $4 \mu \mathrm{g}$ / $\mathrm{mL}$, respectivamente); el estudio de Cai y cols. mostró algunas modificaciones en el ambiente genético de optrA-2, algunos

FIGURA 3. Árbol filogenético de aislamientos de E. faecalis portadores del gen optrA basados en los datos de secuenciación del genoma completo, datos demográficos y concentración inhibitoria mínima al linezolid del aislamiento

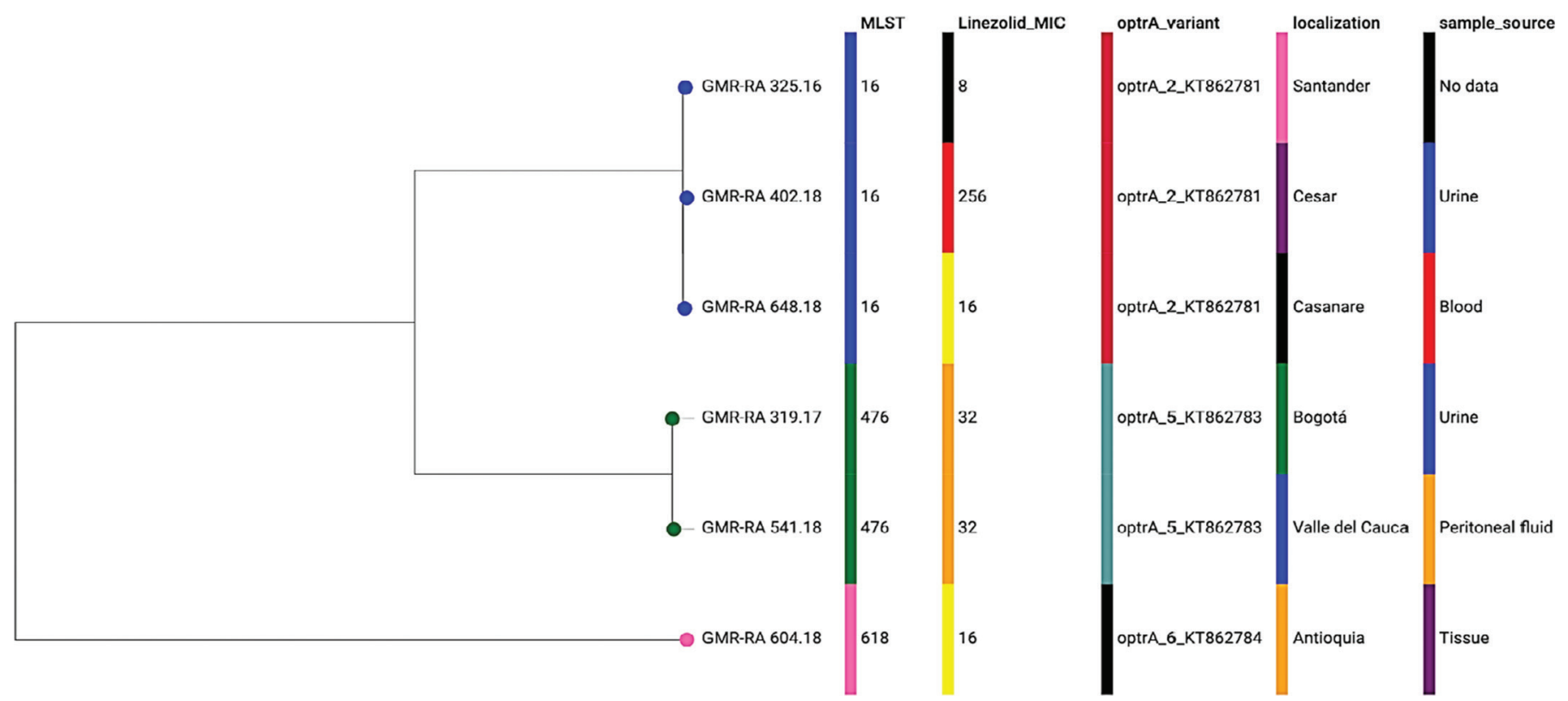


aislamientos pierden el gen $f e x$, mientras que otros presentaban el gen ara $\mathrm{C}$ (regulador transcripcional) corriente arriba de optrA. Sin embargo, los autores sugieren que son necesarios más estudios para aclarar los factores relacionados con la regulación de optrA y los valores de CIM al linezolid (14). En nuestro caso, el ambiente genético de optrA-2 fue diferente al de los aislamientos del estudio de Cai y cols. (14); todos conservaron el gen fex en la misma dirección de optrA y ninguno presentó araC, $\mathrm{y}$ al igual que los investigadores mencionados consideramos necesario profundizar y evaluar los factores asociados con la resistencia al linezolid ( $\mathrm{CIM} \geq 8 \mu \mathrm{g} / \mathrm{mL}$ ) en nuestros aislamientos optrA-2.

En cuanto al entorno genético de optrA, este fue diverso y asociado tanto a plásmido como a cromosoma, lo que sugiere que el mecanismo de diseminación de optrA se relaciona con transferencia horizontal y no con la diseminación de un clon $(16,21)$. Los aislamientos portadores de optrA-2 (ST16) presentaron un segmento común "optrA, fexA e impB", el cual se ha asociado con diferentes plásmidos, en muestras de diversos orígenes y se ha identificado en países como Colombia, Estados Unidos, China, Alemania, España, Escocia, Irlanda, Suecia y Túnez, entre otros $(4,21,32)$. Además del segmento mencionado, dos de los tres aislamientos optrA-2 (GMR-RA 325.16 y GMR-RA 648.18) presentaron los locus tnpA y bin3, relacionados con los transposones Tn3 y Tn552, respectivamente; estos transposones se han asociado con genes de resistencia mientras Tn552 se ha identificado en Enterococcus spp. y Staphylococcus spp., relacionado especialmente con betalactamasas (33); la estructura imp B, fex, optrA, tnp A y bin3 detectada en nuestros aislamientos optrA-2, ha sido identificada en aislamientos de E. faecalis de Japón (pS7316; GenBank: LC499744.1) y Escocia (pWE0438) (32).

Por otra parte, un aislamiento optrA-5 (GMR-RA 541.18; ST476) y otro optrA-6 (GMR-RA 604.18; ST618), presentaron el transposón cromosomal Tn6674-like (miembro de la familia Tn554), considerado uno de los nuevos mecanismos de diseminación de optrA (34), descrito recientemente en aislamientos de diferentes orígenes en países como China, Malasia, Taiwán, Francia y Túnez, $(4,21,34)$, y reportado por primera vez en el continente americano (4). Li y cols. (34) describieron la presencia de formas intermediarias circulares de Tn6674, lo que sugiere que es un transposón activo capaz del escindirse del ADN huésped, formar una estructura circular e integrarse en un nuevo ADN, de igual forma que otros transposones de esta familia (34).

Respecto de la diseminación de optrA en Colombia, se podría considerar un evento policlonal dado que se encontraron tres clases de secuencias tipo (ST). La más prevalente fue ST16 (50\%), la cual es la ST más reportada a nivel mundial en aislamientos positivos para optrA y se ha identificado en aislamientos clínicos de Alemania, China, Dinamarca, Escocia y Tailandia $(4,8,11,16$, 22-24, 32), así como en cerdos en Colombia (21). Igualmente, ST476 se ha identificado en aislamientos humanos $(11,14,21)$ y de animales (11) mientras que, hasta la fecha, ST618 solo se ha reportado en animales (11) y este ST pertenece al CC59 al cual también pertenecen dos de los tres aislamientos reportados en carne cruda en Colombia (16).

Aunque todos los aislamientos optrA positivos de este estudio fueron E. faecalis resistentes al linezolid, cabe resaltar que un estudio que evaluó la estructura poblacional de E. faecium resistente a la vancomicina (EfmRV) en Latinoamérica (35) reportó en Colombia un aislamiento de EfmRV portador de optrA pero sensible al linezolid; este fenómeno se ha descrito previamente en China (14).
A la fecha, en la vigilancia nacional solo se recibe aislamientos no sensibles al linezolid para detección de $\operatorname{optr} A$, por lo cual sería necesario evaluar este gen en nuestros aislamientos de Enterococcus spp. sensibles al linezolid para establecer la prevalencia de optrA en este tipo de aislamientos en Colombia.

En los aislamientos de este estudio, adicional a optrA se identificó una combinación de diferentes determinantes de resistencia a múltiples antimicrobianos (fexA, ermB, lsaA, tet $(M)$, tet $(L) d f r G$ ) y al igual que otros estudios podemos sugerir que la propagación de optrA en diferentes ambientes (comunidad, hospital o ambiente agrícola) puede estar dada posiblemente por la presión selectiva ejercida por antimicrobianos diferentes a las oxazolidinonas $(11,24)$.

En este estudio, el 43\% de los individuos en los que se aisló $E$. faecalis positivo para optrA acudieron a consulta externa. De los nueve casos a los que se les realizó encuesta siete fueron considerados colonizados; esto sugiere que estos individuos son portadores y, de manera similar a otros estudios, no fue posible establecer el factor de riesgo asociado $(14,24)$. Cai y cols. (14) sugieren que estos individuos sanos son portadores comensales de E. faecalis positivos para optrA y esta puede ser una de las formas de propagación de estos patógenos (14). Por otra parte, ocho individuos del estudio se encontraban ubicados en diferentes servicios de hospitalización. De ellos, se revisó la historia clínica de dos niños (26 días y dos meses, respectivamente) y se comprobó que nacieron por cesárea y se encontraban hospitalizados desde su nacimiento, lo cual permite sugerir que presentaron una infección asociada a la atención en salud. Por lo anterior, podemos sugerir que, en Colombia, circula E. faecalis positivo para optrA a nivel tanto comunitario como hospitalario, lo cual complementa los datos de presencia en nuestro país de optrA en alimentos de origen animal y en animales.

Dentro de las limitaciones de este estudio esta no evaluar aislamientos sensibles y no contar con todos los aislamientos a nivel nacional porque la vigilancia es voluntaria. Adicionalmente, no se realizaron ensayos de transferencia de resistencia ni caracterización completa de plásmidos por una limitación técnica del tipo de secuenciación usada.

En conclusión, nuestro estudio describe que en los aislamientos clínicos de $E$. faecalis recibidos en la vigilancia nacional el principal mecanismo de resistencia a linezolid es el gen optrA. Estos aislamientos presentan alta diversidad genética, con diferentes clones y variantes de optrA relacionados con dos tipos de estructuras y diferentes elementos genéticos móviles. Teniendo en cuenta que, en Colombia, optrA se ha identificado en humanos, alimentos y animales es importante reforzar la detección de este mecanismo de resistencia a través de una vigilancia integrada con el fin de comparar datos, establecer dinámicas de transmisión y estrategias de prevención que permitan contener la diseminación de optrA en los diferentes con base en el concepto de "una sola salud".

Contribución de los autores. SYS y CD concibieron el estudio. SYS, JFB, EME, MKR, AMH, MVO planificaron los experimentos. SR y GT realizaron el estudio de campo. Todos los autores analizaron los datos e interpretaron los resultados. SYS, CD, JB, EAM, GT y SR escribieron el manuscrito. Todos los autores revisaron y aprobaron la versión final.

Agradecimientos. A todos los laboratorios de salud pública departamentales y secretarías distritales de salud y sus 
respectivas redes de laboratorios, las cuales hacen parte de la Red Nacional de Laboratorios del Instituto Nacional de Salud y que participan en la Vigilancia Nacional por Laboratorio de la Resistencia a los Antimicrobianos en Microorganismos Causantes de IAAS del INS de Colombia. Al Programa de Entrenamiento de Epidemiología de Campo del Instituto Nacional de Salud de Colombia y al equipo de vigilancia epidemiológica del Departamento de Santander por el apoyo en la caracterización epidemiológica de los casos.

Conflicto de intereses. Ninguno declarado.
Financiamiento. Los reactivos para la caracterización fenotípica y detección del gen optrA utilizados en la vigilancia nacional estuvieron a cargo del Instituto Nacional de Salud. Los reactivos usados en este trabajo han sido parcialmente financiados por la Red PulseNet para América Latina y el Caribe (OPS/OMS).

Declaración. Las opiniones expresadas en este manuscrito son únicamente responsabilidad de los autores y no reflejan necesariamente las de la Revista Panamericana de Salud Pública o la Organización Panamericana de la Salud.

\section{REFERENCIAS}

1. Long KS, Vester B. Resistance to linezolid caused by modifications at its binding site on the ribosome. Antimicrob Agents Chemother. 2012; 56 (2): 603-612. Doi: 10.1128/AAC.05702-11.

2. Rincón S, Panesso D, Díaz L, Carvajal LP, Reyes J, Munita JM et al. Resistencia a antibióticos de última línea en cocos Gram positivos: la era posterior a la vancomicina. Biomedica. 2014; 34 Suppl (1):191208. Doi: $10.1590 /$ S0120-41572014000500022.

3. Hashemian SMR, Farhadi T, Ganjparvar M. Linezolid: a review of its properties, function, and use in critical care. Drug Des Devel Ther. 2018; 12:1759-1767. Doi: 10.2147/DDDT.S164515. eCollection 2018.

4. Deshpande LM, Castanheira M, Flamm RK, Mendes RE. Evolving oxazolidinone resistance mechanisms in a worldwide collection of enterococcal clinical isolates: results from the SENTRY Antimicrobial Surveillance Program. J Antimicrob Chemother. 2018; 73 (9); 2314-22. Doi: 10.1093/jac/dky188.

5. Pfaller MA, Mendes RE, Streit JM, Hogan PA, Flamm RK. Five-year summary of in vitro activity and resistance mechanisms of linezolid against clinically important Gram-positive cocci in the United States from the LEADER Surveillance Program (2011 to 2015). Antimicrob Agent Chemother. 2017; 61 (7): e00609-17. Doi 10.1128/AAC.00609-17.

6. Mendes RE, Deshpande L, Streit JM, Sader HS, Castanheira M, Hogan PA, Flamm RK. ZAAPS programme results for 2016: an activity and spectrum analysis of linezolid using clinical isolates from medical centres in 42 countries. J Antimicrob Chemother. 2018;73(1):1880-7. Doi: 10.1093/jac/dky099

7. Cui L, Wang Y, Lv Y, Wang S, Song Y, Li Y, Liu J et al. Nationwide surveillance of novel oxazolidinone resistance gene optrA in Enterococcus isolates in China from 2004 to 2014. Antimicrob Agent Chemother. 2016;60(12):7490-3. Doi:10.1128/AAC.01256-16.

8. Bender JK, Fleige C, Lange D, Klare I, Werner G. Rapid emergence of highly variable and transferable oxazolidinone and phenicol resistance gene optrA in German Enterococcus spp. clinical isolates. Int J Antimicrob Agents. 2018;52(6):819-27. Doi: 10.1016/j. ijantimicag.2018.09.009

9. Long KS, Poehlsgaard J, Kehrenberg C, Schwarz S, Vester B. The Cfr rRNA methyltransferase confers resistance to phenicols, lincosamides, oxazolidinones, pleuromutilins, and streptogramin A antibiotics. Antimicrob Agents Chemother. 2006;50(7):2500-5. Doi: 10.1128/AAC.00131-06.

10. Antonelli A, D’Andrea MM, Brenciani A, Galeotti CL, Morroni G, Pollini $S$ at al. Characterization of poxtA, a novel phenicoloxazolidinone-tetracycline resistance gene from an MRSA of clinical origin. J Antimicrobial Chemother. 2018;73(7):1763-9. Doi: 10.1093/ $\mathrm{jac} / \mathrm{dky} 088$.

11. Wang Y, Lv Y, Cai J, Stefan S, Langing C, Hu Z et al. A novel gene, optrA, that confers transferable resistance to oxazolidinones and phenicols and its presence in Enterococcus faecalis and Enterococcus faecium of human and animal origin. J Antimicrob Chemother. 2015;70(8):2182-90. Doi: 10.1093/jac/dkv116

12. Sharkey LK, Edwards TA, O’Neill AJ. ABC-F Proteins Mediate Antibiotic Resistance through Ribosomal Protection. MBio. 2016;7(2):e01975-15. doi: 10.1128/mBio.01975-15.

13. Fan R, Li D, Feßler AT, Wu C, Schwarz S, Wang Y. Distribution of optrA and cfr in florfenicol-resistant Staphylococcus sciuri of pig origin. Vet Microbiol. 2017;210:43-8. Doi: 10.1016/j.vetmic.2017.07.030
14. Cai J, Schwarz S, Chi D, Wang Z, Zhang R, Wang Y. Faecal carriage of optrA-positive enterococci in asymptomatic healthy humans in Hangzhou, China. Clin Microbiol Infect. 2019;25(5):630.e1-630.e6. Doi: $10.1016 /$ j.cmi.2018.07.025.

15. Huang J, Sun J, Wu Y, Chen L, Duan D, Wang L. Identification and pathogenicity of an XDR Streptococcus suis isolate that harbours the phenicol-oxazolidinone resistance genes optrA an cfr, and the bacitracin resistance locus bcrABDR. Int J Antimicrob Agents. 2019;54(1):43-8. Doi: 10.1016/j.ijantimicag.2019.04.003

16. Cavaco LM, Bernal JF, Zankari E, Léon M, Hendriksen RS, PerezGutierrez E et al. Detection of linezolid resistance due to the optrA gene in Enterococcus faecalis from poultry meat from the American continent (Colombia). J Antimicrob Chemother. 2017;72(3):678-3. Doi: $10.1093 / \mathrm{jac} / \mathrm{dkw} 490$.

17. Munk P, Knudsen BE, Lukjancenko O, Duarte ASR, Van Gompel $\mathrm{L}$, Luiken REC, et al. Abundance and diversity of the faecal resistome in slaughter pigs and broilers in nine European countries. Nat Microbiol. 2018;3:898-908. Doi: 10.1038/s41564-018-0192-9.

18. Elghaieb H, Tedim AP, Abbassi MS, Novais C, Duarte B et al. From farm to fork: identical clones and Tn6674-like elements linezolidresistant Enterococcus faecalis from food-producing animals and retail meat. J Antimicrob Chemother. 2020;75(1):30-5. Doi: 10.1093/ $\mathrm{jac} / \mathrm{dkz} 419$.

19. Freitas AR, Elghaieb H, León-Sampedro R, Abbassi MS, Novais C, Coque TM et al. Detection of optrA in the African continent (Tunisia) within a mosaic Enterococcus faecalis plasmid from urban wastewaters. J Antimicrob Chemother. 2017;72(12):3245-51. Doi: $10.1093 / \mathrm{jac} / \mathrm{dkx} 321$.

20. Zhao Q, Wang Y, Wang S, Wang Z, Du XD, Jiang H et al. Prevalence and Abundance of Florfenicol and Linezolid Resistance Genes in Soils Adjacent to Swine Feedlots. Sci Rep. 2016;6:32192. Doi: $10.1038 /$ srep32192.

21. Freitas AR, Tedim Ap, Novais C, Lanza VF, Peixe L. Comparative Genomics of Global optrA-carrying Enterococcus faecalis Uncovers a Common Chromosomal Hotspot for optrA Acquisition within a Diversity of Core and Accessory Genomes. Microb Genom. 2020;6(6):1-17. Doi:10.1099/mgen.0.000350

22. Vorobieva V, Roer L, Justesen US, Hansen F, Frimodt-Møller N, Hasman H, Hammerum AM. Detection of the OptrA gene in a clinical ST16 Enterococcus faecalis isolate in Denmark. J Glob Antimicrob Resist. 2017;10:12-3. Doi: 10.1016/j.jgar.2017.05.002.

23. Tsilipounidaki K, Gerontopoulos A, Papagiannitsis C, Penitaki E. First Detection of an optrA-positive, Linezolid-Resistant ST16 Enterococcus faecalis From Human in Greece. New Microbes and New Infect. 2019.;29:100515. Doi: 10.1016/j.nmni.2019.01.010

24. Cámara J, Camoez M, Tubau F, Pujol M, Ayats J, Ardanuy C, et al. Detection of the Novel optrA Gene Among Linezolid-Resistant Enterococci in Barcelona, Spain. Microb Drug Resist. 2019;25(1):8793. Doi: $10.1089 / \mathrm{mdr} .2018 .0028$.

25. Clinical and Laboratory Standard Institute (CLSI). Performance Standards for Antimicrobial Susceptibility Testing; $28^{\text {th }}$ ed. CLSI supplement M100. Wayne: Clinical and Laboratory Standards Institute; 2018.

26. Zankari E, Hasman H, Cosentino S, Vestergaard M, Rasmussen S, Lund $\mathrm{O}$, et al. Identification of acquired antimicrobial resistance 
genes. J Antimicrob Chemother. 2012;67(11): 2640-4. Doi: 10.1093/jac/ dks261.

27. Hasman H, Clausen PTLC, Kaya H, Hansen F, Knudsen JD, Wang $\mathrm{M}$, et al. LRE-Finder, a Web tool for detection of the 23S rRNA mutations and the optrA, cfr, $\operatorname{cfr}(\mathrm{B})$ and poxtA genes encoding linezolid resistance in enterococci from whole-genome sequences. J Antimicrob Chemother. 2019;74(6):1473-6. Doi: 10.1093/jac/dkz092

28. Carattoli A, Zankari E, García-Fernández A, Voldby Larsen M, Lund $\mathrm{O}$, Villa L, et al. In silico detection and typing of plasmids using PlasmidFinder and plasmid multilocus sequence typing. Antimicrob Agents Chemother. 2014; 58(7):3895-3903. Doi: 10.1128/ AAC.02412-14.

29. Seemann T. Prokka: rapid prokaryotic genome annotation. Bioinformatics. 2014;30(14):2068-9. Doi: 10.1093/bioinformatics/btu153

30. Camacho C, Coulouris G, Avagyan V, Ma N, Papadopoulos J, Bealer $\mathrm{K}$, et al. BLAST+: architecture and applications. BMC Bioinformatics. 2008;10:421. doi: 10.1186/1471-2105-10-421

31. Carver T, Berriman M, Tivey A, Patel C, Böhme U, Barrell BG, et al. Artemis and ACT: viewing, annotating and comparing sequences stored in a relational database. Bioinformatics. 2008;24(23):2672-6. Doi: $10.1093 /$ bioinformatics/btn529.

32. McHugh MP, Parcell BJ, Pettigrew KA, Toner G, Khatamzas E, Karcher AM et al. Emergence of optrA-mediated linezolid resistance in multiple lineages and plasmid of Enterococcus faecalis revealed by long read sequencing. bioRxiv. 2020. Doi: 10.1101/ 2020.02.28.969568

33. Partridge SR, Kwong S, Firth N, Jensen S. Mobile Genetic Elements Associated with Antimicrobial Resistance. Clin Microbiol Rev. 2018;31(4): e00088-17. Doi: 10.1128/CMR.00088-17.

34. Li D, Li X-Y, Schwarz S, Yang M, Zhang S-M, et al. Tn6674 is a novel enterococcal optrA-carrying multiresistance transposon of the Tn554 family. Antimicrob Agents Chemother. 2019;63(9):e00809-19. Doi: 10.1128/AAC.00809-19.

35. Rios R, Reyes J, Carvajal P, Rincon S, Panesso D, Echeverry AM et al. Genomic Epidemiology of Vancomycin-Resistant Enterococcus Faecium (VREfm) in Latin America: Revisiting the Global VRE Population Structure. Sci Rep. 2020;10: 5636. Doi: 10.1038/ s41598-020-62371-7

Manuscrito recibido el 3 de noviembre de 2019. Aceptado para su publicación, tras revisión, el 16 de julio de 2020.

\section{National surveillance of clinical isolates of Enterococcus faecalis resistant to linezolid carrying the optrA gene in Colombia, 2014-2019}

ABSTRACT

Keywords
Objective. To describe the epidemiological, phenotypical and genetic characteristics of clinical isolates carrying the optrA gene identified in antimicrobial resistance surveillance by the laboratory of the National Institute of Health of Colombia.

Methods. Between October 2014 and February 2019, 25 isolates of Enterococcus spp. resistant to linezolid were received. Antimicrobial identification and sensitivity were determined using Vitek 2 and the minimum inhibitory concentration (MIC) to linezolid was established with E-test. The optrA gene was detected by PCR, and the genetic diversity of optrA-positive isolates was tested with Diversilab ${ }^{\circledR}$. Six isolates were selected to perform whole genome sequencing.

Results. The optrA gene was confirmed in $23 / 25$ isolates of $E$. faecalis from seven departments in Colombia. The isolates presented a MIC to linezolid between 8 and $>256 \mu \mathrm{g} / \mathrm{mL}$. Typing by Diversilab ${ }^{\oplus}$ showed a wide genetic variability. All the isolates analyzed by whole genome sequencing showed the resistance genes fexA, ermB, IsaA, tet $(M)$, tet $(L)$ and $d f r G$ in addition to optr $A$ and were negative for other mechanisms of resistance to linezolid. Three type sequences and three optrA variants were identified: ST16 (optrA-2), ST476 (optrA-5) and ST618 (optrA-6). The genetic environment of the optrA-2 (ST16) isolates presented the impB, fex, optrA segment, associated with plasmid, while in two isolates (optrA-6 and optrA-5) the transferable chromosomal element Tn6674-like was found.

Conclusion. OptrA-positive clinical isolates present a high genetic diversity, with different optrA clones and variants related to two types of structures and different mobile genetic elements.

Enterococcus faecalis; linezolid; drug resistance, microbial; Colombia. 